\title{
Importance of Standards in Biomedical Device and Its Role in Strengthening the Healthcare Sector
}

\author{
Gajjala Sumana*, Rajesh and D. K. Aswal \\ Council of Scientific and Industrial Research-National Physical Laboratory, New Delhi, India
}

OPEN ACCESS

Edited by:

Pratima R. Solanki, Jawaharlal Nehru University, India

Reviewed by:

Anil Kumar

National Institute of Immunology

(NII), India

Lakshmi G. B. V. S,

Jawaharlal Nehru University, India

${ }^{*}$ Correspondence:

Gajjala Sumana

sumanagajjala@gmail.com

Specialty section: This article was submitted to Biomedical Nanotechnology, a section of the journal Frontiers in Nanotechnology

Received: 29 October 2020 Accepted: 16 March 2021 Published: 13 April 2021

Citation: Sumana G, Rajesh and Aswal DK (2021) Importance of Standards in Biomedical Device and Its Role in Strengthening the Healthcare Sector.

Front. Nanotechnol. 3:622804. doi: 10.3389/fnano.2021.622804
Biomedical Metrology group of CSIR-NPL (Council of Scientific and Industrial ResearchNational Physical Laboratory), India is contributing significantly for establishing National Biomedical equipment standardswhich are traceable to primary standards to cater the quality control regulation in Health sector. This initiative has great impact on the health policy and Nation's economy as well. The laboratory has recently established biomedical equipment standard facility and disseminated the services to Industries, stake holders, hospitals and accredited laboratories. Although, the laboratory is successfully providing biomedical equipment calibration services for last 2 years, the sensitization of the information on the recently established metrological capabilities is required, which has great implications in implementation of India's New medical Device rule 2017 effectively. The present paper describes the summary on the significant achievements and role of National Physical Laboratory in supporting the Indian medical device regulations.

Keywords: biomedical, metrology, quality, infrastructure, calibration, growth

\section{INTRODUCTION}

Ministry of Health and Family welfare of Government of India has published "New Medical Device and in vitro diagnostic regulations in 2017" and as per the new regulations, ministry's undertaken organization, central drugs standard control organization has issued medical device rule 2017 and are being implemented in India with effect from January 1, $2018^{1}$ Medical device regulations accentuate the major requirement of quality control and periodic calibration checks of medical equipment to minimize the medical device errors. In the medical equipment market, the quality control is very important for the correct diagnosis and correct treatment of the patients. Precise and accurate measurements are vital for taking appropriate medical decisions. Whereas, un-calibrated medical devices lead to critical errors in equipment leading to wrong diagnosis, medical device failures causing causality and deaths. Therefore, regular calibration of the biomedical equipment to assure the accurate and precise measurements which are traceable to national standards is very crucial in implementing National Quality health infrastructure in Health sector. In this context, CSIR-NPL, Indian National Metrological Institute is serving the country by providing calibration facilities traceable to National standards to support the quality regulation of Medical equipment.

\section{IMPLICATIONS OF BIOMEDICAL METROLOGY IN HEALTHCARE SECTOR}

Recently in September 2019, World Health Organization has estimated that nearly 134 million

${ }^{1}$ https://cdsco.gov.in/opencms/opencms/en/Medical-Device-Diagnostics/Medical-Device-Diagnostics/. 


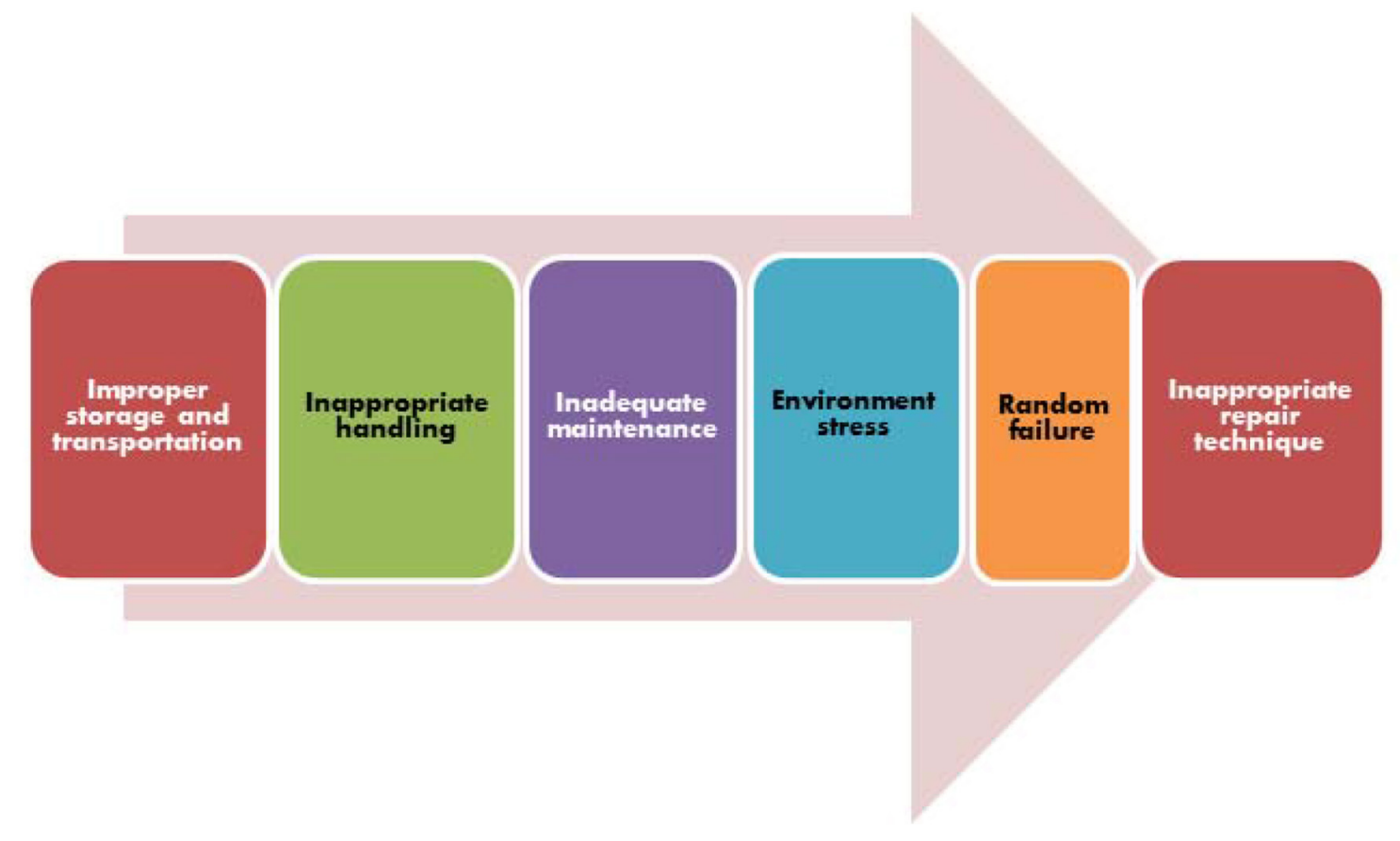

FIGURE 1 | Common causes for biomedical equipment error.

medical errors occur in hospitals in low- and middle-income countries including the biomedical equipment errors ${ }^{2}$ The common biomedical equipment errors are depicted in Figure 1. As per the Harvard University report 2013, Medical errors are the third leading cause of death in the United States with causalities as high as 0.4 million (Jha et al., 2013). According to WHO estimation $\sim 80 \%$ failures of medical equipment can be prevented by their proper maintenance, calibration and periodic inspections. Estimates indicate that among various reasons for the medical equipment errors, inadequate maintenance alone accounts for about $60 \%$ and $20 \%$ are accounted to inappropriate handling, environmental stress and wear-out etc. WHO (2011) as depicted in (Figure 2).

Besides healthcare, the quality control in medical devices and its implementation have great implications in the National economy as well. "Make in India" sector survey on Medical Devices estimated that the Indian medical device market has great potential and its market value is estimated to increase steeply to USD 50 billion by 2025. Currently, the market value of Biomedical and Healthcare industry is around USD 5.2 billion, which is one of the largest economic sectors and is contributing greatly in National economy. Presently, India is the fourth largest medical device manufacturing country in Asia with an average turnover of Rs 450-500 million ${ }^{3}$ Implementing corrective measures such as regulating the medical quality

\footnotetext{
${ }^{2}$ https://www.who.int/news-room/fact-sheets/detail/patient-safety.

${ }^{3} \mathrm{https} / /$ www.makeinindia.com/article/-/v/sector-survey-medical-devices.
}

infrastructure, regular accreditation of biomedical equipment, development of standards, adopting the standard procedural protocols in healthcare institutions are a few steps that have great impact in the industrial development of Biomedical devices. In this context, CSIR-National Physical Laboratory is playing a pivotal role in healthcare sector by developing equipment standards and providing calibration as per the internationally accepted technical standards for boosting up the domestic biomedical industries and calibration laboratories.

\section{REGULATORY RECOMMENDATIONS}

Medical device regulations are regulated by the National authority and it varies from country to country. In India, the medical device regulations are regulated by Central Drugs Standard Control Organization. According to Indian regulatory Authority, the medical equipment has to satisfy adequate safety, performance and quality norms as per ISO-17025 guidelines. Medical devices have been classified into four classes as per the risk based qualification viz. class A (low risk), class B (low moderate risk), class $\mathrm{C}$ (moderate risk), and class $\mathrm{D}$ (high risk).

As per the Indian regulatory authorities, the biomedical electrical equipment should conform to technical standards as per the guidelines of Electro-technical Commission (IEC-60601) (Costa Monteiro and Leon, 2015), International Organization for Standardization (ISO-17025) ${ }^{4}$ and Bureau of Indian standards (BIS) published Indian standards and procedures. Therefore, as per the BIS documentation and IEC standards and country's legal

\footnotetext{
${ }^{4}$ https://www.iso.org/standard/65529.html.
} 


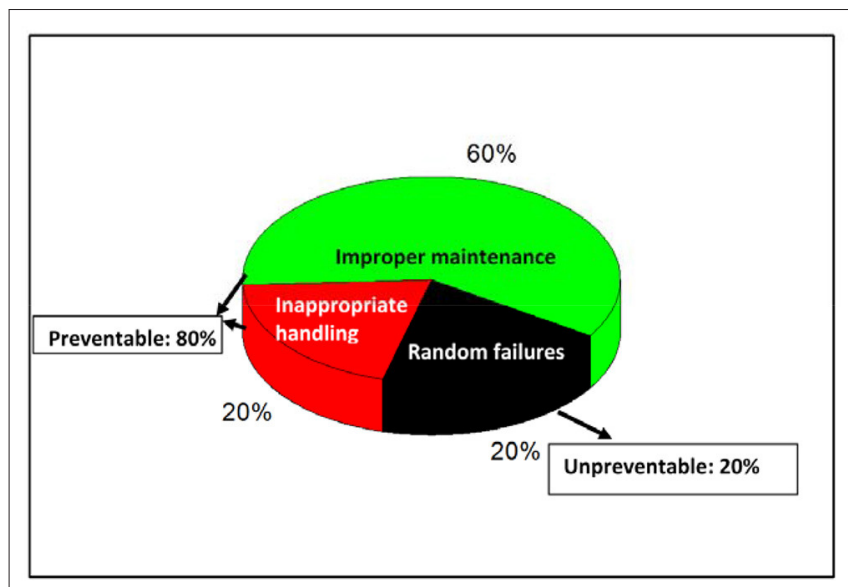

FIGURE 2 | Biomedical equipment errors.

metrology requirements, CSIR-National physical laboratory is working toward the creation and maintenance of the biomedical equipment standards.

\section{BIOMEDICAL METROLOGY PROGRAM AT CSIR-NATIONAL PHYSICAL LABORATORY}

CSIR-National Physical Laboratory, Indian National Metrological Institute, has taken initiative to create and maintain national standards in biomedical instrumentation with traceability to National Standards. In September 2018, CSIR-NPL has established apex level biomedical equipment standard for Defibrillator and Defibrillator analyzer where the measurements are traceable to National standards (Rajesh et al., 2019). CSIR-NPL is providing apex level calibration facilities for clinical thermometers, blood pressure monitors and magnetic resonance etc. Efforts are being made to develop and disseminate

\section{REFERENCES}

Costa Monteiro, E., and Leon, L. F. (2015). Metrological reliability of medical devices. J. Phys. Conf. Ser. 588. doi: 10.1088/1742-6596/588/1/012032

Jha, A. K., Larizgoitia, I., Audera-Lopez, C., Prasopa-Plaizier, M., Waters, H., and Bates, D. W. (2013). The global burden of unsafe medical care: analytic modelling of observational studies. BMJ Qual. Saf. 22, 809-815. doi: 10.1136/bmjqs-2012-001748

Rajesh, V. K. T., Sumana, G., Agarwal, V.V., Ojha, V. N., and Aswal, D. K. (2019). CSIR-NPL establishes an apex-level calibration facility for defibrillator analyzer and defibrillator machine. Current Sci. 117:25. doi: $10.18520 / \mathrm{cs} / \mathrm{v} 117 / \mathrm{i} 2 / 179-180$

WHO (2011). WHO Medical equipment maintenance programme overview. WHO Medical device technical series Available online at: https://apps.who. the standards for biomedical equipment such as Electrical safety analyzer, Infusion and Syringe pumps, dialysis equipment and Incubators etc. The development of Biomedical equipment standards for calibration of Medical devices and dissemination of these standards to industries, secondary laboratories and hospitals will have a huge impact on National healthcare sector in reducing the health complications due to medical device errors.

Keeping the above importance of the issue in view, CSIRNational Physical Laboratory through biomedical metrology program is working on the following objectives:

- Establishing the National standards of Biomedical equipment comparable to International standards through continuous Research and Development.

- Dissemination of Apex level calibration standards as per National and International standards to hospitals, clinical labs, biomedical industries and institutes and other stakeholders.

- To support the regulatory bodies in the context of newly enacted “Medical Device Rule 2017” effective from 1st Jan 2018 and ISO 17025:2017.

- Training on Testing and Calibration of Biomedical Instruments.

\section{CONCLUSIONS}

CSIR-National Physical Laboratory of India is playing a critical role in implementing the quality control of biomedical equipment by providing calibration traceable to primary standards. The importance of biomedical metrology and its implications in National Healthcare and country economy are highlighted in the present manuscript.

\section{AUTHOR CONTRIBUTIONS}

All Authors have contributed for the onset of the biomedical metrology activities at NPL.

int/iris/bitstream/handle/10665/44587/9789241501538_eng.pdf/jsessionid= 156EC3788A40D9EB8B7E9CD5EAA741BB? sequence $=1$

Conflict of Interest: The authors declare that the research was conducted in the absence of any commercial or financial relationships that could be construed as a potential conflict of interest.

Copyright (c) 2021 Sumana, Rajesh and Aswal. This is an open-access article distributed under the terms of the Creative Commons Attribution License (CC BY). The use, distribution or reproduction in other forums is permitted, provided the original author(s) and the copyright owner(s) are credited and that the original publication in this journal is cited, in accordance with accepted academic practice. No use, distribution or reproduction is permitted which does not comply with these terms. 